\title{
Glycoprotein Structure of Microconidial Invertase from Fusarium oxysporum
}

\author{
Masafumi Nishizawa and Yoshiharu MaruYama \\ Department of Agricultural Chemistry, The University of Tokyo, \\ Tokyo 113, Japan \\ Received June 30, 1980
}

\begin{abstract}
An examination of glycopeptide fractions obtained by proteolytic digestion of microconidial invertase isozyme (P-2) of Fusarium oxysporum revealed two classes of polysaccharide chains differing in size and composition. The nature of the carbohydrate to protein linkages was studied, and P-2 had both $N$-glycosidic bondings between asparagine and $N$-acetylglucosamine, and $O$ glycosidic bondings between serine or threonine and mannose. Based on these findings, a tentative glycoprotein structure of P-2 is discussed.
\end{abstract}

During the study of invertase from Fusarium oxysporum, we found that the organism can produce two invertase isozymes, $\mathrm{P}-1$ and $\mathrm{P}-2^{1)}$ both of which are glycoproteins containing mannose as their major sugar component. ${ }^{2}$ The P-1 isozyme exists in mycelia and microconidia, whereas the P-2 form exists exclusively in microconidia. ${ }^{3)}$ Unlike the invertases from Saccharomyces cerevisiae and Neurospora crassa which are glycoproteins with mannose but no glucose or galactose in their carbohydrate moieties, ${ }^{4)}$ the Fusarium invertases contain carbohydrate units with all three types of hexoses. ${ }^{2)}$

In the past decade, remarkable advances in our understanding of glycoprotein structure, biosynthesis, and function have been made. For example, in glycoproteins which have carbohydrate chains linked to peptides through $\quad N$-acetylglucosaminylasparaginyl bonds, a 'core' oligosaccharide has been found that is apparently common among eukaryotic cells from yeast to mammals. ${ }^{5)}$ Such an oligosaccharide structure has not been demonstrated in any glycoproteins from Fusarium. Moreover, it is of interest to determine how glucose and galactose residues are built up in the polysaccharide chains of Fusarium invertases. These sugars are not common in yeast and Neurospora invertases though in- vertase secreted by Candida utilis contains a small amount of glucose. ${ }^{6}$ )

With regard to the properties of polysaccharide chains of glycoprotein-enzymes (glycoenzymes), they are suggested to function as a protector to stabilize the conformation of the enzymes by providing resistance to proteolysis $^{7)}$ and/or to denaturation. ${ }^{8,9)}$ Their precise properties, however, have not been clarified as yet. We consider that the two Fusarium invertase isozymes, $\mathrm{P}-1$ and $\mathrm{P}-2$, have a potential utility in studying the function of polysaccharide chains of glycoenzymes because $\mathrm{P}-2$ is much more heat-stable than P$1^{10)}$ although both are glycoenzymes. Thus, to study glycoprotein structure of Fusarium invertases will provide more information on functions of polysaccharide chains in glycoenzymes as well as their structure.

In this paper, we studied glycoprotein structure of P-2 invertase, and clarified that there are two groups of polysaccharide chains which differ both in size and carbohydrate composition, and that both $\mathrm{N}$-glycosidic and $O$-glycosidic bondings are involved in carbohydrate to protein linkages.

\section{MATERIALS AND METHODS}

Materials. Pronase E (Streptomyces griseus, 45,000 U 
per g) was obtained from Kaken Kagaku Co. Endo- $\beta-N$ acetylglucosaminidase $\mathrm{H}$ (Streptomyces plicatus), $\beta-N$ acetylhexosaminidase (Turbo cornutus), and $\beta$-galactosidase (Charonia lampus) were from Seikagaku Kogyo. $\alpha$ Mannosidase (Canavalia ensiformis), $\beta$-glucosidase (sweet almond), and neuraminidase (Clostridium perfringens) were from Boehringer-Mannheim. Bio-gel P-100 was purchased from Bio-Rad. Sephadex G-50, G-100 and G200 were from Pharmacia. Other chemicals were of the best grade available.

Enzyme purification. P-2 invertase from Fusarium oxysporum f. sp. gladioli IFO 5894 were prepared according to the method described previously. ${ }^{2)}$ The preparation used in these experiments appeared homogeneous on the bases of polyacrylamide gel electrophoresis, column chromatography, and ultracentrifugation.

Assays. Protein was determined by the method of Lowry, et al.,${ }^{11)}$ and total carbohydrates were determined by phenol-sulfuric acid method. ${ }^{12)}$

Gas-liquid chromatography (GLC). For analysis, sugars were converted to acetates or alditol-acetates. ${ }^{13}$ ) The alditol-acetate derivatives were prepared and analyzed as described in a previous paper. ${ }^{2)}$ Acetate derivatives were prepared as follows: samples were hydrolyzed with $1 \mathrm{~N}$ methanol-hydrochloric acid at $110^{\circ} \mathrm{C}$ for $24 \mathrm{hr}$. The hydrolysates were neutralized with silver carbonate, and sugars were extracted with methanol, dried, and then acetylated using acetic anhydride in pyridine. The acetyl derivatives were analyzed with a Shimadzu GC-4CM gas chromatograph equipped with a flame ionization detector and glass columns packed with $3 \%$ ECNSS-M on Gaschrome Q (100 120 mesh, Wako Pure Chem. Ind.). A Shimadzu E-1A Chromatopak was used to calculate peak areas. Myo-inositol was used as an internal standard.

Amino acid analyses. Amino acid composition was determined with a Hitachi 835 amino acid analyzer. Samples were hydrolyzed with $6 \mathrm{~N}$ hydrochloric acid for $24 \mathrm{hr}$ at $110^{\circ} \mathrm{C}$ under nitrogen, and then the hydrochloric acid was removed by evaporation. The hydrolysates were dissolved in $100 \mu \mathrm{l}$ of $0.02 \mathrm{~N}$ hydrochloric acid, and $50 \mu \mathrm{l}$ of aliquot of the solution was injected into the analyzer. Norleucine was used as an internal standard.

Pronase digestion of $P-2$ invertase. A $19.3 \mathrm{mg}$ portion of P-2 invertase was digested with Pronase $E$ in $1 \mathrm{ml}$ of $0.05 \mathrm{M}$ borate buffer, $\mathrm{pH} 7.8$, containing $0.0015 \mathrm{M}$ calcium chloride for $96 \mathrm{hr}$ at $37^{\circ} \mathrm{C}$ in the presence of a small amount of toluene. ${ }^{14)}$ Prior to the addition of Pronase, the invertase solution was heated to $100^{\circ} \mathrm{C}$ for $3 \mathrm{~min}$. After cooling, $5 \mathrm{U}$ of Pronase was added to the digestion solution and mixed. The mixture was incubated at $37^{\circ} \mathrm{C}$. At 24 -hr intervals, $5 \mathrm{U}$ portions of Pronase were added.
After the incubation, the digests were lyophilized, dissolved in $1 \mathrm{ml}$ of $0.1 \mathrm{M}$ pyridine-acetate buffer, $\mathrm{pH} 5.0$, and fractionated on a Sephadex G-50 column $(2.5 \times 60 \mathrm{~cm})$. Fractions were assayed for carbohydrates and amino acids.

Endo- $\beta$ - $N$-acetylglucosaminidase $H$ digestion of $P$-2, A 5 mg portion of P- 2 was incubated with $50 \mathrm{mU}$ of endo- $\beta-N-$ acetylglucosaminidase $\mathrm{H}$ in $0.5 \mathrm{ml}$ of $0.1 \mathrm{M}$ sodium citrate buffer, $\mathrm{pH} 5.5^{15)}$ for $24 \mathrm{hr}$ at $37^{\circ} \mathrm{C}$. After the incubation, the digest was lyophilized, dissolved in $0.1 \mathrm{M}$ pyridineacetate buffer, $\mathrm{pH}$ 5.0, and fractionated with a Sephadex G-200 column $(2.5 \times 70 \mathrm{~cm})$ to separate digestion products.

$\alpha-$ Mannosidase and exoglycosidases digestion of glycopeptide fractions. Two glycopeptide fractions were obtained after proteolytic digestion of P-2 (see 'Results'). The fraction which contained mainly mannose was incubated with $75 \mathrm{mU}$ of $\alpha$-mannosidase in $1 \mathrm{ml}$ of $0.1 \mathrm{M}$ sodium citrate buffer, $\mathrm{pH} 4.5$, containing $2 \mathrm{~mm}$ zinc acetate. ${ }^{16)}$ The other glycopeptide fraction was incubated with $20 \mathrm{mU}$ each of neuraminidase, $\beta$-galactosidase, $\beta$ glucosidase, and $\beta-N$-acetylhexosaminidase in $1 \mathrm{ml}$ of $0.1 \mathrm{M}$ sodium citrate buffer, $\mathrm{pH} 5.0$. After incubating for $48 \mathrm{hr}$ at $37^{\circ} \mathrm{C}$, each digest was lyophilized, dissolved in $0.1 \mathrm{M}$ pyridine-acetate buffer, $\mathrm{pH} 5.0$, and subjected to chromatography on a Sephadex G-50 column $(2.5 \times 60$ $\mathrm{cm})$ to separate digestion products.

Mild alkaline- $\mathrm{NaBH}_{4}$ treatment $^{17)}$ of $\mathrm{P}-2$. A $10 \mathrm{mg}$ of $\mathrm{P}-$ 2 was incubated in $1 \mathrm{ml}$ of $0.1 \mathrm{~N}$ sodium hydroxide containing $0.3 \mathrm{M}$ sodium borohydride for $168 \mathrm{hr}$ at $37^{\circ} \mathrm{C}$ in the presence of a small amount of toluene. After the incubation, the solution was neutralized with glacial acetic acid, and dialyzed against deionized water for $48 \mathrm{hr}$. After a portion of the dialysate was removed to analyze amino acid composition, the remaining material was lyophilized and dissolved in $0.1 \mathrm{M}$ pyridine-acetate buffer, $\mathrm{pH} 5.0$. The solution was applied to a column of Bio-gel P-100 $(2.5 \times 60$ $\mathrm{cm}$ ), which was eluted with the same buffer. Mild alkalinesodium borohydride treatment of the glycopeptide fractions obtained after proteolytic digestion (see 'Results') was performed in $0.2 \mathrm{ml}$ of $0.1 \mathrm{~N}$ sodium hydroxide containing $12 \mu \mathrm{g}$ (protein) of the glycopeptide fraction and $0.3 \mathrm{M}$ sodium borohydride. After incubating for $120 \mathrm{hr}$ at $37^{\circ} \mathrm{C}$, the solution was neutralized and subjected to amino acid analysis.

\section{RESULTS}

\section{Isolation of glycopeptides from Pronase digest of $P-2$}

Chromatography of the Pronase digest of $\mathrm{P}$ 2 on a Sephadex G-50 column gave the elution profile shown in Fig. 1. Two peaks containing 


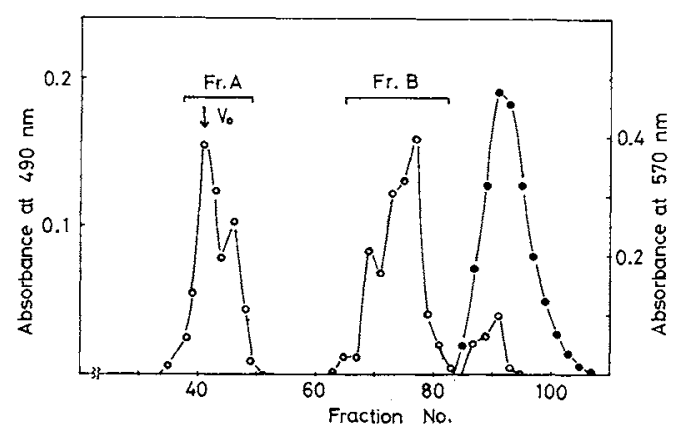

Fig. 1. Gel Filtration on Sephadex G-50 of Pronase Digests of P-2 Invertase.

Column $(2.5 \times 60 \mathrm{~cm})$ was previously equilibrated and eluted with $0.1 \mathrm{M}$ pyridine-acetate buffer, $\mathrm{pH} 5.0$. Each fraction volume was $2.5 \mathrm{ml}$. Elution profile shows total carbohydrates by phenol-sulfuric acid method ${ }^{12)}$ $(\mathrm{O}-\mathrm{O})$ and amino acids by ninhydrin reaction ${ }^{25}$ ) (-), expressed as absorbance at 490 and $540 \mathrm{~nm}$, respectively. An arrow indicates the void volume $(\mathrm{Vo})$ of the column.

carbohydrates were obtained. The material in each peak was collected separately, lyophilized, and assayed for protein and carbohydrate contents. The material in the first peak eluted from the column, Fraction A, had the ratio of protein to carbohydrate, $1: 10.5$. In the second peak, Fraction $B$, the ratio was $1: 2.9$. In the native $\mathrm{P}-2$ enzyme, the ratio was $3.7: 1 .^{2)}$ The overall recovery of carbohydrate in Fractions $\mathrm{A}$ and $\mathrm{B}$ was $86 \%$.

\section{Carbohydrate and amino acid compositions of glycopeptides}

The carbohydrate and the amino acid compositions of the two glycopeptide fractions were determined. Figure 2 shows the gas chromatograms of sugars of Fractions A and B. Fraction A contained $N$-acetylglucosamine (GlcNAc), $N$-acetylgalactosamine (GalNAc), mannose, glucose and galactose, whereas Fraction B contained mainly GlcNAc and mannose. Table I summarizes the compositions of carbohydrate and amino acids in the two fractions. In Fraction $\mathrm{A}$, the content of mannose ( $45 \%$ of total sugar) was almost equal to the sum of glucose and galactose $(43 \%)$, and the content of GlcNAc was only

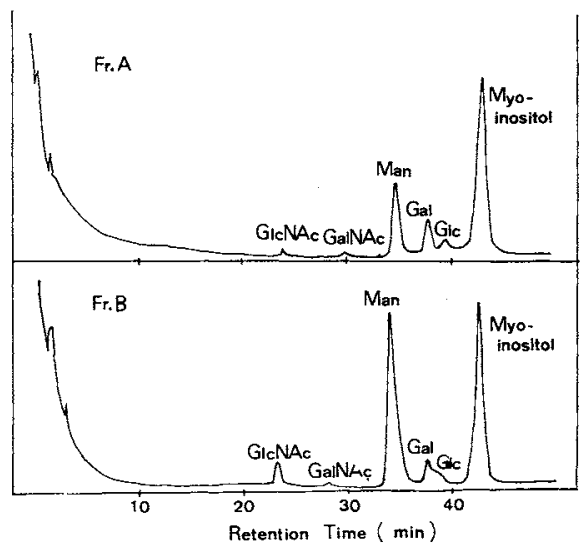

FIG. 2. Gas-Liquid Chromatography of Sugar Components of Fractions $\mathrm{A}$ and $\mathrm{B}$.

Sugars were analyzed as alditol-acetate derivatives. Glass columns were packed with $0.75 \%$ HiEFF-IBP, $0.25 \%$ EGSS-X and $0.1 \%$ PDEA on Gaschrome $(60 \sim 80$ mesh). ${ }^{26)}$ GLC conditions were as follows: column temp., initial $130^{\circ} \mathrm{C}$, final $180^{\circ} \mathrm{C}$, programming rate; $1{ }^{\circ} \mathrm{C} / \mathrm{min} \cdot$ detector temp, $280^{\circ} \mathrm{C}$; injector temp., $250^{\circ} \mathrm{C} ; \mathrm{N}_{2}$ flow rate, $50 \mathrm{ml} / \mathrm{min}$. Each peak was identified as indicated in the figures by its retention time relative to the internal standard. GlcNAc, $N$-acetylglucosamine; GalNAc, $N$-acetylgalactosamine; Man, mannose; Gal, galactose; and Glc, glucose.

$5 \%$. In Fraction $\mathrm{B}$, the major components were mannose $(73 \%)$ and GicNAc $(20 \%)$.

Table I also shows amino acid compositions of the two glycopeptide fractions and native $\mathrm{P}$ 2 expressed as molar ratios taking aspartic acid as one. The predominant amino acids in both glycopeptide fractions are aspartic acid, threonine, and serine, suggesting these amino acids are localized close to the site of the linkage of carbohydrate to protein in the intact enzyme.

Fr. A appeared in the void volume when applied to either a Sephadex G-50 column (Fig. 1) or a Sephadex G-100 column (data not shown), but it was fractionated by a Sephadex G-200 column giving three major carbohydrate-containing fractions, which were named Frs. A-I, A-II, and A-III according to the order of elution (Fig. 3). Table II summarizes the carbohydrate compositions of the three fractions. The glucose content was highest in Fraction A-I (45\%), decreasing to 
Table I. Amino ACID AND Carbohydrate Compositions of Pronase Digest OF P-2 Invertase

\begin{tabular}{lccc}
\hline & Fr. A & Fr. B & Native P-2 ${ }^{2)}$ \\
\hline Amino acid & & & \\
Aspartic acid & $1.00^{*}$ & 1.00 & 1.00 \\
Threonine & 1.35 & 0.83 & 0.57 \\
Serine & 1.39 & 0.65 & 0.58 \\
Glutamic acid & 0.98 & 0.27 & 0.55 \\
Proline & 0.38 & 0.23 & 0.32 \\
Glycine & 1.15 & 0.60 & 0.58 \\
Alanine & 1.16 & 0.19 & 0.47 \\
Valine & 0.60 & 0.17 & 0.41 \\
Isoleucine & 0.26 & 0.12 & 0.34 \\
Leucine & 0.46 & 0.08 & 0.50 \\
Tyrosine & 0.27 & 0.06 & 0.32 \\
Phenylalanine & 0.13 & 0.02 & 0.35 \\
Lysine & 0.42 & 0.11 & 0.38 \\
Histidine & 0.31 & 0.09 & 0.21 \\
Arginine & 0.30 & 0.05 & 0.23 \\
Carbohydrate & & & \\
GlcNAc & $5 \%$ & $20 \%$ & $14 \%$ \\
GalNAc & $6 \%$ & $3 \%$ & $8 \%$ \\
Mannose & $45 \%$ & $73 \%$ & $69 \%$ \\
Glucose & $11 \%$ & $4 \% * *$ & $7 \%$ \\
Galactose & $32 \%$ & & $2 \%$ \\
\hline
\end{tabular}

* Each amino acid content is expressed as the molar ratio taking aspartic acid as one.

** Glucose and galactose in Fr. B were not separated by GLC.

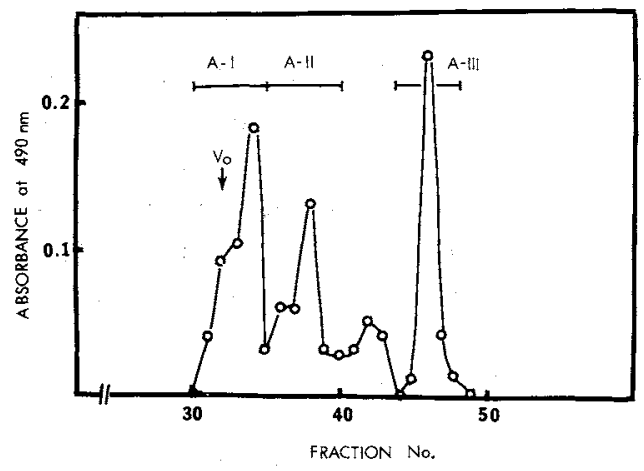

FIG. 3. Gel Filtration on Sephadex G-200 of Fraction A Glycopeptide.

Column $(2 \times 80 \mathrm{~cm})$ was previously equilibrated and eluted with $0.1 \mathrm{M}$ pyridine-acetate buffer, pH 5.0. Each fraction volume was $2.5 \mathrm{ml}$. Elution profile shows total carbohydrates expressed as absorbance at $490 \mathrm{~nm}$ by phenol-sulfuric acid method. ${ }^{12)}$ Fraction numbers 30 to 35,36 to 40 , and 44 to 48 were collected as Fractions A-I, A-II and A-III.
TABle II. Carbohydrate Compositions of FRactions A-I, A-II, AND A-III

\begin{tabular}{llrr}
\hline Sugars & Fr. A-I & \multicolumn{1}{c}{ A-II } & A-III \\
\hline GlcNAc & N.D.* & $7.0 \%$ & N.D. \\
GalNAc & N.D. & $3.0 \%$ & N.D. \\
Mannose & $32.7 \%$ & $44.0 \%$ & $51.7 \%$ \\
Galactose & $22.0 \%$ & $25.2 \%$ & $31.7 \%$ \\
Glucose & $45.3 \%$ & $19.8 \%$ & $16.7 \%$ \\
\hline
\end{tabular}

* N.D., not detected.

$20 \%$ in $\mathrm{A}-\mathrm{II}$ and $17 \%$ in A-III. Conversely, the content of mannose increased in the later fractions (A-I, 33\%; A-II, 44\%; and A-III, $52 \%$ ). The content of galactose was almost constant in the three fractions. If the three fractions were separated by gel filtration according to their sizes, this result implies that a larger polysaccharide chain is richer in glucose residues.

\section{Exoglycosidases digestion}

From the results described above, we suspected that the carbohydrate chains of P-2 have the following structure: GIcNAc and mannose residues form core units which are analogous to Asn-GlcNAc-type cores reported in other glycoproteins ${ }^{5)}$; the polysaccharide chains of Fr. B would correspond to these core units, whereas the polysaccharide chains of Fr. A contain core units to which glucose and galactose residues are attached.

To obtain evidence for this tentative structure, we first tried to digest each glycopeptide fraction with exoglycosidases. If we could obtain polysaccharide chains which correspond to those of Fr. B by digestion of Fr. A with glucosidase and galactosidase, our conclusion would be strengthened. For this experiment, we used $\beta$-glucosidase and $\beta$-galactosidase because $\beta$-configuration of glucoside and galactoside predominates in the glycoproteins so far investigated. And neuraminidase was added to remove terminal $N$-acetylneuramic acid, if any, which could interfere with the action of other glycosidases.

Figure 4 shows the elution profile obtained by chromatography of the exoglycosidases- 


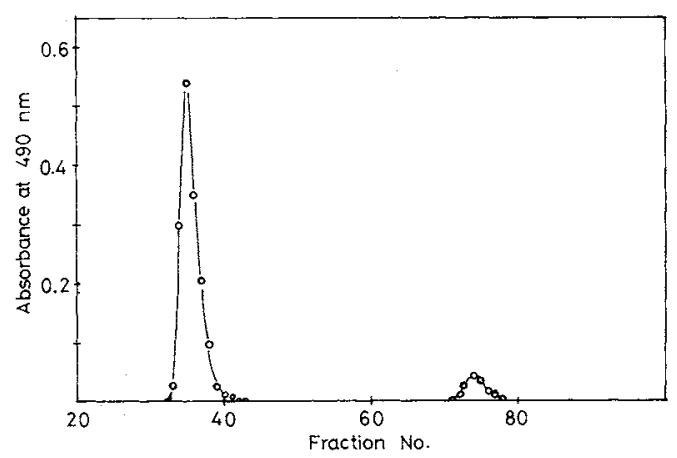

FIG. 4. Gel Filtration on Sephadex G-50 of Exoglycosidases Digest of Fraction A Glycopeptide.

Conditions were the same as the legend to Fig. 1. Fraction numbers 32 to 40 and 70 to 78 were collected separately as carbohydrate-containing fractions.

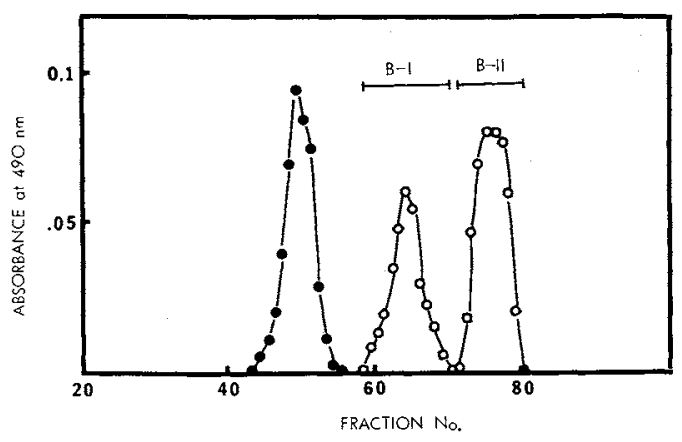

FiG. 5. Gel Filtration on Sephadex G-50 of $\alpha$ Mannosidase Digest of Fraction B Glycopeptide.

Conditions were the same as the legend to Fig. 1. Elution profile shows total carbohydrates of the digests $(\mathrm{O}-\mathrm{O})$, and of native Fraction B (--) obtained by a separate experiment. Fraction numbers 58 to 70 and 71 to 80 were collected separately, and named Fractions B-I and B-II, respectively.

digest on Sephadex G-50. The major carbohydrate fraction was eluted in the void volume, and a small amount of carbohydrate was eluted from fraction 70 to 78 . Assay for the sugar content of each peak showed that although some glucose, galactose and GlcNAc had been released, the digestion of Fr. A had not proceeded to completion. Fr. A was then digested with the same mixture of exoglycosidases supplemented by $\alpha$-mannosidase, but the result was not markedly changed (data not shown).
Table III. Carbohydrate Compositions of $\alpha$-Mannosidase Digest of FR. B GLyCOPEPTIDE

\begin{tabular}{lcc}
\hline \multicolumn{1}{c}{ Sugars } & Fr. B-I & \multicolumn{1}{c}{ B-II } \\
\hline Total carbohydrate & $495 \mu \mathrm{g}$ & \multicolumn{1}{c}{$470 \mu \mathrm{g}$} \\
GleNAc & $54.4 \%$ & N.D.* \\
GalNAc & $0.7 \%$ & N.D. \\
Mannose & $43.1 \%$ & $98.6 \%$ \\
Galactose & $1.0 \%$ & $0.9 \%$ \\
Glucose & $0.5 \%$ & $0.5 \%$ \\
\hline
\end{tabular}

* N.D., not detected.

When Fr. B was digested with $\alpha$-mannosidase and the digest was fractionated on a column of Sephadex G-50, two fractions containing carbohydrate were obtained; they were named Frs. B-I and B-II according to the order of elution (Fig. 5). As shown in Table III, the carbohydrate contents of Frs. B-I and B-II were almost equal to each other, and the sugar released by $\alpha$-mannosidase (Fr. B-II) was mannose alone. This result indicates that Fr. B contains $\alpha$-mannan which is susceptible to jack bean $\alpha$-mannosidase digestion.

\section{Nature of protein-carbohydrate linkage}

There are two major bondings, $\mathrm{N}$-glycosidic bondings (Asn-GlcNAc) and $O$-glycosidic bondings (e.g. Ser/Thr-Man), which link polysaccharide chains to peptides in fungal glycoprotein molecule. To study the nature of protein-carbohydrate linkages in $\mathrm{P}-2$, we tried both digestion of P-2 with endo- $\beta$ - $N$-acetylglucosaminidase, which is known to cleave the di- $N$-acetylchitobiose unit linked to asparagine, ${ }^{18)}$ and treatment of $\mathrm{P}-2$ with mild alkali in the presence of sodium borohydride $(\beta$ elimination reaction).

a) Endo- $\beta$ - $N$-acetylglucosaminidase digestion. Figure 6 shows the elution profile resulting from the chromatography of the endo- $\beta-N$-acetylglucosaminidase $\mathrm{H}$ digest of native P-2 on Sephadex G-200. Polysaccharide chains of P-2 were partially removed by this digestive enzyme, and they were eluted from the column between fraction 26 (void volume) and 37 . The fractions containing protein and 


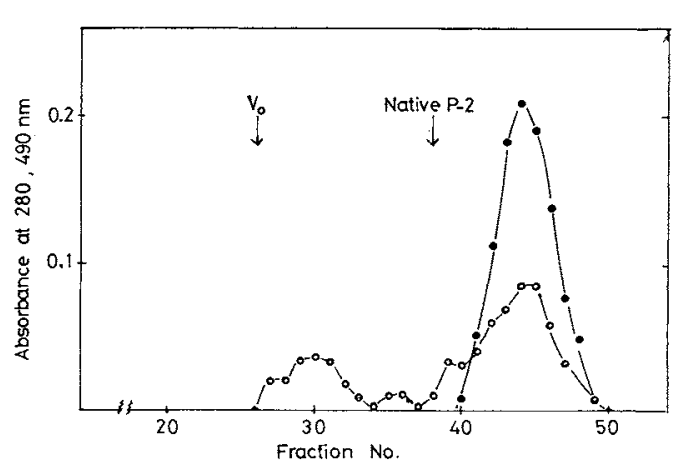

FIG. 6. Gel Filtration on Sephadex G-200 of Endo- $\beta$ $N$-Acetylglucosaminidase $\mathrm{H}$ Digest of P-2 Invertase.

Column $(2.5 \times 70 \mathrm{~cm})$ was previously equilibrated and eluted with $0.1 \mathrm{M}$ pyridine-acetate buffer, $\mathrm{pH} 5.0$. Each fraction volume was $2.5 \mathrm{ml}$. Elution profile shows total carbohydrates expressed as absorbance at $490 \mathrm{~nm}$ (O-O), and protein by absorbance at $280 \mathrm{~nm}$ (---O). Arrows indicate void volume of the column ( $\mathrm{Vo})$ and elution peak of native P-2. Fraction numbers 26 to 37 , and 41 to 48 were collected separately.

carbohydrate which could correspond to partially degraded $\mathbf{P}-2$ were eluted later than those carbohydrate-containing fractions. Invertase activity was coincident with the elution profile of protein (data not shown). The two groups of fractions, Frs. 26 to 37 and 41 to 48 , were collected as polysaccharide chains removed by endo- $\beta$ - $N$-acetylglucosaminidase $\mathrm{H}$ and as modified P-2, respectively. Their carbohydrate contents were $210 \mu \mathrm{g}$ and $430 \mu \mathrm{g}$, respectively, and the removed carbohydrate contained mannose, galactose and glucose. These results indicate that $\mathrm{P}-2$ has polysaccharide chains which are susceptible to endo- $\beta-N$-acetylglucosaminidase $\mathrm{H}$, namely, $N$-glycosidically linked polysaccharide chains.

b) Mild alkali-NaBH$H_{4}$ treatment of $\mathrm{P}-2$. When glycoprotein which contains $O$ glycosidic bondings are treated with mild alkali in the presence of sodium borohydride, these bonds are cleaved through the $\beta$-elimination reaction, and the reaction products are reduced, that is, the sugar once involved in the linkage is converted to corresponding alcohol, and the amino acids, serine and threonine, are converted to alanine and $\alpha$-amino butyric acid, respectively ${ }^{17)}$ We tried this treatment on $\mathrm{P}-2$

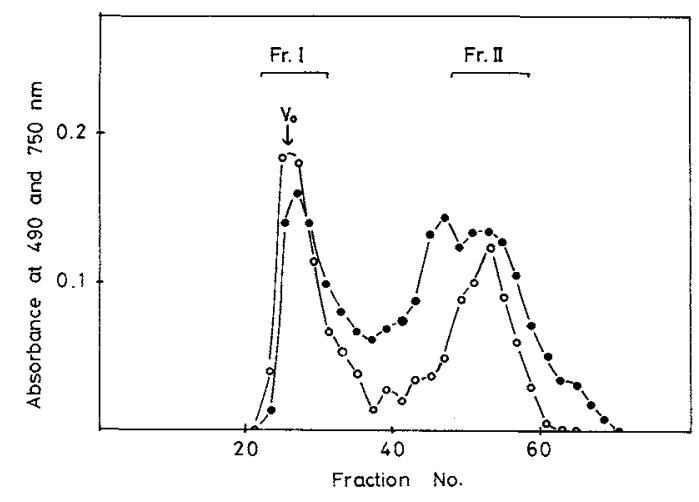

FIG. 7. Gel Filtration in Bio-Gel P-100 of Mild Alkali$\mathrm{NaBH}_{4}$ Treated P-2 Invertase.

Conditions were the same as the legend to Fig. 1. Elution profile shows total carbohydrates $(0--O)$ and protein by the method of Lowry et al. ${ }^{11)}$ expressed as absorbance at $750 \mathrm{~nm}$ (---) Fraction numbers 22 to 30 and 48 to 58 were combined separately and named Fractions I and II, respectively.

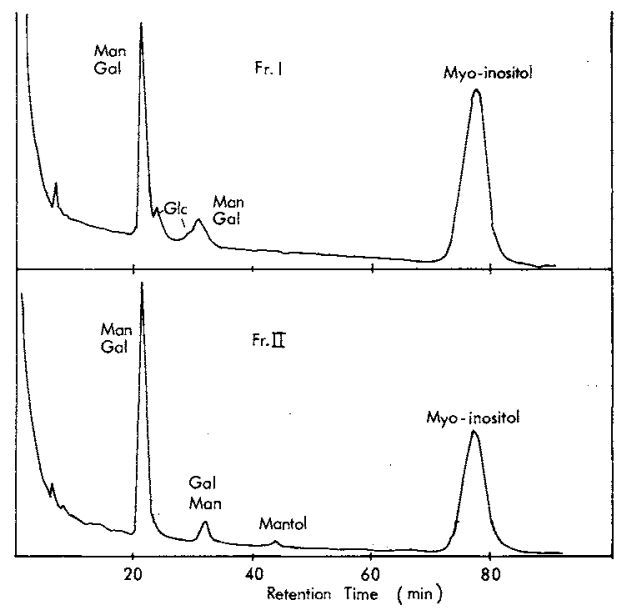

FIG. 8. Gas-Liquid Chromatography of Sugar Components in Fractions I and II.

Sugars of Fractions I and II obtained after mild alkalisodium borohydride treatment of P-2 were analyzed as acetate derivatives. The conditions of GLC were as follows: column temp., $180^{\circ} \mathrm{C}$; detector temp., $280^{\circ} \mathrm{C}$; injector temp., $280^{\circ} \mathrm{C} ; \mathrm{N}_{2}$ flow rate, $50 \mathrm{ml} / \mathrm{min}$. Each peak was identified as indicated in the figures by its retention time relative to the internal standard: Man, mannose; Gal, galactose; Glc, glucose; and Mantol, mannitol. Under these conditions, the peaks of mannose and galactose overlapped.

and determined if a sugar alcohol was generated, and if a change of amino acid com- 
position occurred.

Figure 7 shows the chromatography of alkaline-sodium borohydride-treated $\mathrm{P}-2$ on bio-gel P-100. Two carbohydrate-containing fractions were obtained and named Fractions I and II according to the elution order. The carbohydrate compositions of Fractions I and II were examined by GLC as acetates. As shown in Fig. 8, Fraction I contained mannose, galactose and glucose, and Fraction II contained mannose and mannitol. No amino sugars could be detected as acetates. The reason for this is not clear. Mannitol was not detected in carbohydrate moiety of native $\mathrm{P}-2$ when its carbohydrate composition was examined by GLC as acetyl derivatives (data not shown) or as $O$-trimethylsilyl derivatives. ${ }^{2}$ This result indicates that mannose is one of the linkage sugars.

Table IV summarizes the results of analysis of amino acid composition. After treatment with alkali-sodium borohydride, the contents of serine and threonine decreased, that of alanine increased, and $\alpha$-amino butyric acid was generated when compared with their values before treatment. The contents of aspartic acid, glycine and leucine, on the other hand, did not change significantly, indicating serine and threonine residues were affected specifically by this treatment. The two glycopeptide fractions (Fractions A and B) obtained after proteolytic digestion of P-2 were treated with alkali-sodium borohydride in order to confirm the existence of $O$-glycosidic bondings. As shown in Table V, decrease of serine and threonine, and increase of alanine were observed with both fractions. These results indicate that serine and threonine are at linkage sites between carbohydrate and protein. Taken together with the result of sugar analysis, they indicate that Ser/Thr-Man-type $O$-glycosidic bondings exist in P-2.

Thus, we have clarified that P-2 invertase has both $N$-glycosidic and $O$-glycosidic bondings in the linkage between carbohydrate and protein.

\section{DISCUSSION}

The evidence presented in this paper in-

Table IV. EfFect of Alkaline Sodium BOROHYDRIDE TREATMENT ON P-2 INVERTASE

\begin{tabular}{lcc}
\hline \multicolumn{1}{c}{ Amino acid } & $\begin{array}{c}\text { Before } \\
\text { treatement } \\
(\mu \mathrm{mol} / \mathrm{mg} \text { of protein })\end{array}$ & $\begin{array}{c}\text { After } \\
\text { treatment* }\end{array}$ \\
\hline Threonine & 0.76 & 0.57 \\
Serine & 0.71 & 0.43 \\
Alanine & 0.62 & 0.64 \\
$\alpha$-Amino butyric acid & 0 & 0.07 \\
Aspartic acid & 1.29 & 1.32 \\
Glycine & 0.77 & 0.76 \\
Leucine & 0.66 & 0.57 \\
\hline
\end{tabular}

* The values of this column were corrected to original amount of invertase on the basis of protein content of the solution removed from the dialysate.

Table V. Effect of Alkaline Sodium Borohydride Treatment on Glycopeptide Fractions Obtained by Proteolytic Digestion of P-2 Invertase

\begin{tabular}{llcccc}
\hline \multirow{2}{*}{ Amino acid } & \multicolumn{2}{c}{ Fr. A } & & \multicolumn{2}{c}{ Fr. B } \\
\cline { 3 - 4 } \cline { 5 - 6 } & Before & After & & Before & After \\
\hline Aspartic acid & $1.00^{*}$ & 1.00 & & 1.00 & 1.00 \\
Threonine & 1.35 & 0.55 & & 0.83 & 0.68 \\
Serine & 1.39 & 1.12 & & 0.66 & 0.55 \\
Alanine & 1.16 & 1.36 & & 0.19 & 0.37 \\
Glutamic acid & 0.98 & 0.97 & & 0.37 & 0.39 \\
Glycine & 1.15 & 1.23 & & 0.60 & 0.62 \\
Isoleucine & 0.26 & 0.25 & 0.12 & 0.16 \\
\hline
\end{tabular}

* Each amino acid content is expressed as the molar ratio taking aspartic acid as one. 
dicates that microconidial invertase $(\mathrm{P}-2)$ of Fusarium is a glycoprotein which contains mainly two groups of polysaccharide chains. The two groups are different from each other in size and carbohydrate composition. The apparently large chains (polysaccharide chains of Fr. A) contain GlcNAc, GalNAc, mannose, glucose, and galactose, whereas the small chains (those of Fr. B) contain GIcNAc and mannose. This conclusion is supported by the different carbohydrate compositions between Frs. I and II shown in Figs. 7 and 8. The two fractions were obtained after treatment of $\mathrm{P}-2$ with alkali-sodium borohydride. Fr. I contained mannose, glucose, and galactose as neutral sugars, whereas Fr. II contained mannose only as neutral sugar.

The heterogeneity of polysaccharide chains of glycoproteins is commonly observed and is considered to be due either to some degradation or to incomplete biosynthesis. Also in yeast invertase, the heterogeneity with respect to size is observed in the mannan chains. ${ }^{19)} \mathrm{It}$ is characteristic of Fusarium P-2 invertase, however, that the heterogeneity is not only in size but in carbohydrate composition of polysaccharide chains.

Recent studies on the glycoprotein structure of yeast invertase have clarified that the structure of the polysaccharide chains is approximately the same with that of $N$ glycosidically linked mannoprotein of the organism. ${ }^{20}$ This suggests that the biosynthesis of fungal polysaccharide and the carbohydrate moiety of glycoenzyme could have common pathways. Though we have no information about polysaccharides of the Fusarium we used in these experiments, the polysaccharide from $F$. moniliforme (Gibberella fujikuori) is reported to contain mannose, glucose, galactose, glucuronic acid, and protein. ${ }^{21)}$ This could support the heterogeneous sugar composition of polysaccharide chains of P-2 invertase.

With regard to the nature of linkages between carbohydrate and protein, we showed that both $\mathrm{N}$-glycosidic bonds of the AsnGlcNAc type and $O$-glycosidic bonds of the
Ser/Thr-Man type exist in P-2 by digestion with endo- $\beta$ - $N$-acetylghucosaminidase $\mathrm{H}$ and with mild alkali-sodium borohydride treatment. Yeast invertase contains $N$-glycosidic bonds only, ${ }^{18)}$ and Neurospora crassa invertase is reported to contain $O$-glycosidic bonds and possibly $N$-glycosidic bonds. ${ }^{22)}$

The polysaccharide chains of glycoenzymes are thought to protect the enzyme from proteases $^{6}$ ) and/or to stabilize the enzyme conformation against denaturation. ${ }^{7}$ During the course of this work, we observed that P-2 which had not received heat treatment prior to pronase digestion was not digested sufficiently (data not shown), and that P-2 which had some polysaccharide chains removed by endo$\beta$ - $N$-acetylglucosaminidase $\mathrm{H}$ showed less heat-stability than native $\mathrm{P}-2$ (unpublished observation). These observations support the functions of polysaccharide chains proposed previously. In addition, P-2 modified by endo$\beta$ - $N$-acetylglucosaminidase $\mathrm{H}$ digestion still possessed invertase activity, indicating the polysaccharide chains removed from P-2 by the digestive enzyme have no direct relation to invertase activity.

Based on the assumption that the glycopeptide fractions were separated by gel filtration according to their sizes, we drew a tentative glycoprotein structure of P-2 invertase as follows: polysaccharide chains composed of GlcNAc and mannose or of mannose alone are linked to peptide chain through $\mathrm{N}$-glycosidic or $O$-glycosidic bondings, respectively. Glucose and galactose residues are attachd to some of these inner chains to form large chains. Thus, two groups of polysacccharide chains which are different both in size and composition are formed. Though insufficient digestion of the glycopeptides with the mixture of endoglycosidases makes this structure remain still speculative, the observation that jack bean $\alpha$-mannosidase could digest Fr. B but not Fr. A suggests $\alpha$-mannosidase-susceptible mannan chains in Fr. A, if any, are not accessible to the enzyme, that is, are shielded by glucose and galactose residues.

Recent studies on structure and biosynthesis 
of glycoproteins have revealed that AsnGlcNAc-type glycoproteins from various organisms have common structure ${ }^{5)}$ and that their biosynthesis proceeds in an ordered manner. ${ }^{23)}$ These findings could make random sequence of GlcNAc, mannose, glucose and galactose residues in polysaccharide chains of Fr. A less possible, and could indirectly support our speculative structure.

However, jack been $\alpha$-mannosidase can digest $S$. cerevisiae mannan by only $5 \%$, suggesting that Fr. B glycopeptide has different structure from the yeast mannan. Moreover, our speculative structure implies that the $O$-glycosidically linked mannan chain could form a large chain through the attachment of glucose and galactose residues to it. But there are no reports, so far, describing that $O$-glycosidically linked chains could form or function like Asn-GlcNAc-type 'core' structure to which outer chains are attached. $S$. cerevisiae mannoprotein contains both $\mathrm{N}$ glycosidic and $O$-glycosidic bondings, and the $N$-glycosidically linked chains form the core structure, whereas the $O$-glycosidically linked chains contain only a few mannose residues. ${ }^{24)}$ With regard to the structure of the heterogeneous polysaccharide chains, we suspect it to be highly branched because polysaccharide chains removed from P-2 by endo- $\beta$ - $N$-acetylglucosaminidase $\mathrm{H}$ were eluted faster than partially degraded P-2 and even native P-2 (Fig. 6). This abnormal behavior was observed in three separate experiments, and similar behavior was also observed in the chromatography of Fr. A on Sephadex G-200 (Fig. 3). We consider that highly branched structure of the polysaccharide chains could cause such behavior.

The proposed glycoprotein structure of P-2 invertase, although purely speculative, provides a potential model for investigating the biosynthesis of heterogeneous polysaccharide. Detailed structure of polysaccharide chains of $\mathrm{P}-2$ is now under investigation, and will provide more information on structure, biosynthesis and function of polysaccharide chains of glycoenzymes.

\section{REFERENCES}

1) Y. Maruyama and K. Onodera, J. Gen. Appl. Microbiol., 25, 361 (1979).

2) M. Nishizawa, Y. Maruyama and M. Nakamura, Agric. Biol. Chem., 44, 489 (1980).

3) M. Nishizawa and Y. Maruyama, J. Bacteriol, 139, 657 (1979).

4) J. O. Lampen "The Enzyme," Vol. V, ed. by P. D. Boyer, Academic Press Inc., New York, N.Y., 1971, pp. $291 \sim 305$.

5) R. Kornfeld and S. Kornfeld, Annu. Rev. Biochem., 45, 217 (1976).

6) M. Iizuka, H. Chiura and T. Yamamoto, Agric. Biol. Chem., 42, 1207 (1978).

7) J. W. Coffey and C. Deduve, J. Biol. Chem., 243, 3255 (1968).

8) W. N. Arnold, Biochem. Biophys. Acta, 178, 347 (1969).

9) M. Iizuka and T. Yamamoto, Agric. Biol. Chem., 43, 217 (1979).

10) Y. Tashiro, $\mathrm{Ph} . \mathrm{D}$. Thesis, The University of Tokyo $(1970)$.

11) O. H. Lowry, N. J. Rosebrough, A. L. Farr and R. J. Randall, J. Biol. Chem., 193, 265 (1951).

12) M. Dubois, K. A. Gilles, J. K. Hamilton, P. A. Rebers and F. Smith, Anal. Chem., 28, 350 (1956).

13) W. H. Porter, Anal. Chem., 63, 27 (1975).

14) R. G. Spiro, "Methods in Enzymology," Vol. VIII, ed. by S. P. Colowick and N. O. Kaplan, Academic Press Inc., New York, N.Y., 1966, p. 28.

15) A. L. Tarentino, T. H. Plummer, Jr. and F. Maley, $J$. Biol. Chem., 249, 818 (1974).

16) Y. L. Li, J. Biol. Chem., 241, 1010 (1966).

17) A. Gottschalk, "Glycoproteins," Part A, ed. by A. Gottschalk, Elsevier Publishing Co., Amsterdam, 1972, p. 470.

18) A. L. Tarentino and F. Maley, J. Biol. Chem., 249, 811 (1974).

19) N. P. Neumann and J. O. Lampen, Biochem., 8, 3552 (1969).

20) L. Lehle, R. E. Gordon and C. E. Ballou, J. Biol. Chem., 254, 12209 (1979).

21) I. R. Siddiqui and G. A. Adams, Can. J. Chem,, 39, 1683 (1961)

22) Y. Tashiro and J. R. Trevithick, Can. J. Biochem., 55, 249 (1977).

23) I. Tabas and S. Kornfeld, J. Biol. Chem., 253, 7779 (1978).

24) T. Nakajima and C. E. Ballou, J. Biol. Chem., 249, 7679 (1974).

25) E. W. Yemm and E. C. Cocking, Analyst, 80, 209 (1955).

26) W. F. Lehnhardt and R. J. Winzler, J. Chromatogr., 34, 471 (1968). 